
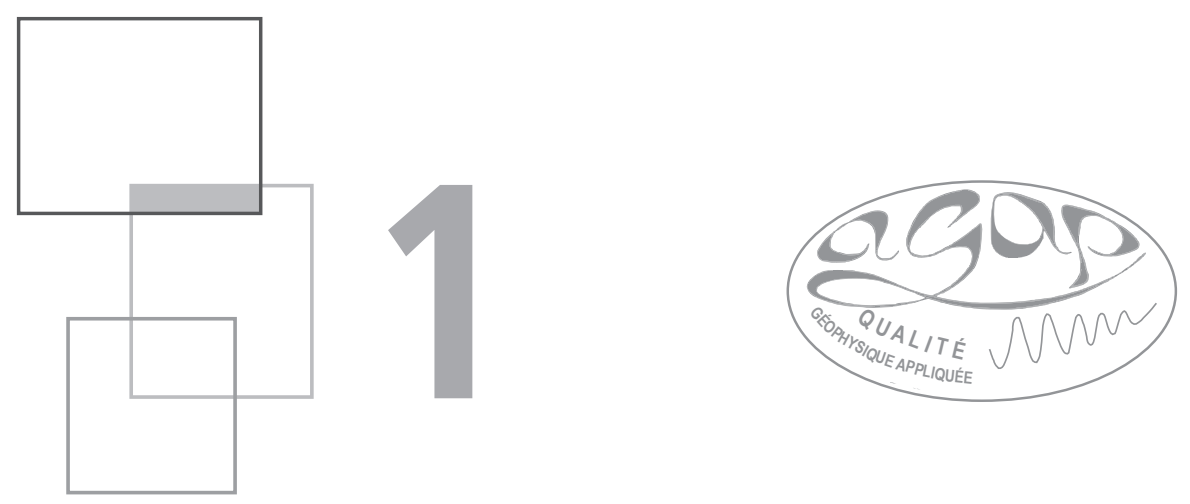

\title{
Wave propagation
}

\section{J.-L. Mari}

Seismic prospecting consists of the generation of very low-amplitude artificial earthquakes at predetermined times and positions. The seismic disturbances generated by a seismic source are recorded by a seismic receiver spread. The acquisition geometry is defined by the distribution of the source spread and the receiver spread.

The following elements are needed to observe the propagation of seismic, acoustic or elastic waves:

1. A source spread. The source is a device capable of producing a deformation in a medium. In land acquisition, it can be an explosive charge (dynamite), a weight dropper or a vibrator. In marine acquisition, it can be an air gun, a sparker or a vibrator. Seismic energy radiated by the source is split between body waves (compressional and shear waves) and surface waves.

2. A physical medium defined by its geometric and mechanical characteristics. Here we consider the geological formations defined by the following mechanical properties:

- Propagation velocity of the compressional $P$-waves in the rock: $\mathrm{V}_{\mathrm{P}}$ (expressed in $\mathrm{m} / \mathrm{s}$ ),

This chapter of Seismic Imaging: a practical approach is published under Open Source Creative Commons License CC-BY-NC-ND allowing non-commercial use, distribution, reproduction of the text, via any medium, provided the source is cited.

(C) EDP Sciences, 2019

DOI: 10.1051/978-2-7598-2351-2.c003 
- Propagation velocity of the shear $S$-waves in the rock: $\mathrm{V}_{\mathrm{S}}$ (expressed in $\mathrm{m} / \mathrm{s}$ ),

- Density $\rho$ (expressed in $\mathrm{g} / \mathrm{cm}^{3}$ or $\mathrm{kg} / \mathrm{m}^{3}$ ),

- Quality factor Q which characterizes the ability of the rock to absorb seismic energy: a higher value indicates lower absorption of seismic energy. Sedimentary rocks have a $\mathrm{Q}$ value ranging from about 10 to several hundred.

3. An elastic deformation of the medium after the initial shaking caused by the source. A deformation is considered elastic when the medium returns to its original state after the causes of deformation have disappeared, i.e. when the medium has not been damaged by the wave passing through it.

4. A receiver spread. Which must be capable of recording the deformations generated by the source after propagation in the geological medium:

- Either by variations in the displacement, velocity or acceleration of particles (geophones, accelerometers),

- Or by pressure variations (hydrophones).

Table 1.1 Seismic velocities and densities, and mechanical moduli (after Lavergne, 1989).

\begin{tabular}{|c|c|c|c|}
\hline $\begin{array}{l}\text { Type of rock } \\
\text { or medium }\end{array}$ & $\begin{array}{l}P \text {-velocity } \\
V_{P}(\mathrm{~m} / \mathrm{s})\end{array}$ & $\begin{array}{l}S \text {-velocity } \\
V_{S}(\mathrm{~m} / \mathrm{s})\end{array}$ & $\begin{array}{l}\text { Density } \\
\rho\left(\mathrm{g} / \mathrm{cm}^{3}\right)\end{array}$ \\
\hline Weathered rocks & $300-700$ & $100-300$ & $1.7-2.4$ \\
\hline Dry sands & $400-1200$ & $100-500$ & $1.5-1.7$ \\
\hline Wet sands & $1500-4000$ & $400-1200$ & $1.9-2.1$ \\
\hline Clay & $1100-2500$ & $200-800$ & $2.0-2.4$ \\
\hline Marl/shale & $2000-3000$ & $750-1500$ & $2.1-2.6$ \\
\hline Sandstone & $3000-4500$ & $1200-2800$ & $2.1-2.4$ \\
\hline Limestone & $3500-6000$ & $2000-3300$ & $2.4-2.7$ \\
\hline Chalk & $2300-2600$ & $1100-1300$ & $1.8-2.3$ \\
\hline Salt & $4500-5500$ & $2500-3100$ & $2.1-2.3$ \\
\hline Anhydrite & $4000-5500$ & $2200-3100$ & $2.9-3.0$ \\
\hline Dolomite & $3500-6500$ & $1900-3600$ & $2.5-2.9$ \\
\hline Granite & $4500-6000$ & $2500-3300$ & $2.5-2.7$ \\
\hline Basalt & $5000-6000$ & $2800-3400$ & $2.7-3.1$ \\
\hline Coal & $2200-2700$ & $1000-1400$ & $1.3-1.8$ \\
\hline Water & $1450-1500$ & - & 1 \\
\hline Ice & $3400-3800$ & $1700-1900$ & 0.9 \\
\hline Oil & $1200-1250$ & - & $0.6-0.9$ \\
\hline \multicolumn{4}{|c|}{ First Lamé parameter $\quad \lambda=\rho\left(V_{P}^{2}-2 V_{S}^{2}\right)$} \\
\hline \multicolumn{4}{|c|}{ Shear modulus (or second Lamé parameter) $\quad \mu=\rho V_{S}^{2}$} \\
\hline \multicolumn{4}{|l|}{ Poisson's coefficient } \\
\hline \multicolumn{4}{|c|}{ Young's modulus $\quad E=\rho V_{P}^{2} \frac{(1-2 \sigma)(1+\sigma)}{1-\sigma}$} \\
\hline Bulk modulus $K=$ & $=\rho\left(V_{P}^{2}-\frac{4}{3} V_{S}^{2}\right)$ & & \\
\hline
\end{tabular}


Table 1 shows the range of values of propagation velocities, $V_{P}$ and $V_{S}$, and the densities of principal rock types. It also gives the expressions of the main mechanical modules (Poisson's coefficient, Young's modulus...).

\subsection{Seismic wave equation}

In seismic prospecting, the energy generated by the seismic source is relatively weak and the medium can be considered as elastic, obeying Hooke's laws. For small deformations, each stress tensor $\left(\sigma_{i, j}\right)$ is a linear combination of the elements of the deformation tensor $\left(\varepsilon_{\mathrm{i}, \mathrm{j}}\right)$. The constants of proportionality for a homogeneous and isotropic medium are Lamé's constants $\lambda$ and $\mu$. The parameter $\mu$ is termed the shear modulus. The displacements $\mathrm{U}_{\mathrm{i}}$ (components of the displacement vector) that are observable at all points within the medium and, particularly, on the surface are solutions to the wave equation. In a three-dimensional rectilinear frame of reference $\left(\mathrm{x}_{\mathrm{i}}, \mathrm{i}=1\right.$ to 3$)$ the wave propagation in the $\mathrm{x}$-direction is written as:

$$
\rho \frac{\partial^{2} U_{i}}{\partial t^{2}}=\sum_{j=1}^{3} \frac{\partial}{\partial x_{j}}\left(\sigma_{i, j}\right)
$$

with:

$$
\begin{aligned}
& \sigma_{i, j}=\lambda\left(\sum_{k=1}^{3} \varepsilon_{k, k}\right)\left(\delta_{i, j}\right)+2 \mu\left(\varepsilon_{i, j}\right) \\
& \varepsilon_{i, j}=\frac{1}{2}\left(\frac{\partial U_{i}}{\partial x_{j}}+\frac{\partial U_{j}}{\partial x_{i}}\right)
\end{aligned}
$$

stress strain

where $\delta_{i, j}$ are the Kronecker symbols:

$$
\delta_{i, j}= \begin{cases}1 & i=j \\ 0 & i \neq j\end{cases}
$$

For each element of the stress tensor $\left(\sigma_{\mathrm{i}, \mathrm{j}}\right)$ the first index $\mathrm{i}$ indicates the stress component in the reference system $\left(x_{i}, i=1\right.$ to 3$)$; the second index $\mathrm{j}$ is the surface undergoing the stress, the surface being defined by its normal in the reference system.

All waves (body waves and surface waves) are solutions to the wave equation. The compressional $P$-waves correspond to longitudinal vibrations which, at every point in the medium, have a particle motion parallel to the direction of propagation (Figure 1.1-a). The propagation velocity for compressional waves is equal to:

$$
\mathrm{V}_{\mathrm{P}}^{2}=(\lambda+2 \mu) / \rho
$$

The shear $S$-waves correspond to transverse vibrations which, at every point in the medium, have a particle motion perpendicular to the direction of propagation (Figure 1.1-b and c). The propagation velocity for shear waves is equal to:

$$
\mathrm{V}_{\mathrm{S}}^{2}=\mu / \rho
$$


A
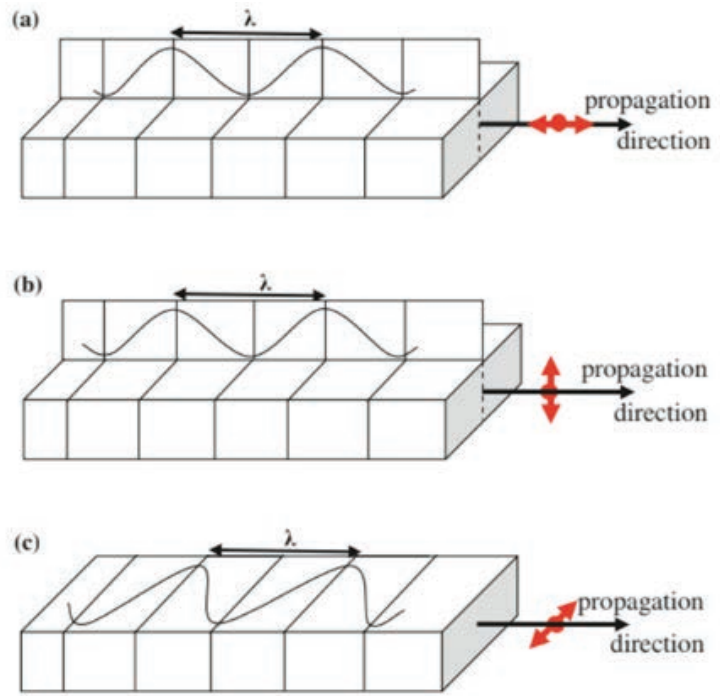

B

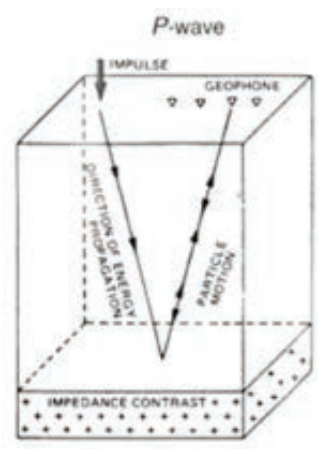

(a)

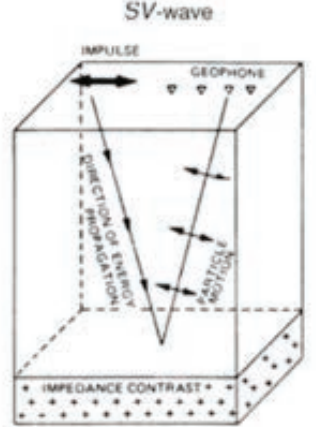

(b)

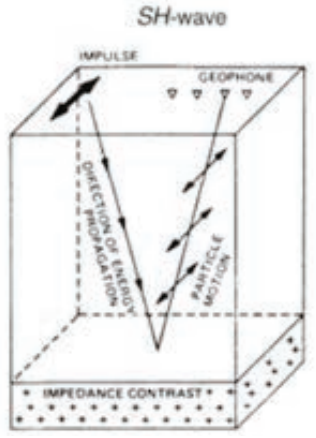

(c)

Figure 1.1 Schematic representation of the particle displacement induced by bodywaves traveling in a solid medium, $(\lambda)$ is the wavelength: $A$ - plane bodywave (a) P-wave, (b) SV-wave, (c) SH-wave. (Adapted from Alsadi, 2017); $B$ - propagation and vibration directions for body waves (a) P-wave, (b) SV-wave, (c) SH-wave (after Ensley, 1985). 
Shear waves do not propagate in fluids. The ratio $\mathrm{V}_{\mathrm{P}} / \mathrm{V}_{\mathrm{S}}$ is independent of the formation density so allows the calculation of Poisson's ratio which is used to differentiate unconsolidated rocks (Poisson's $>0.35$ ) from consolidated rocks (Figure 1.2).

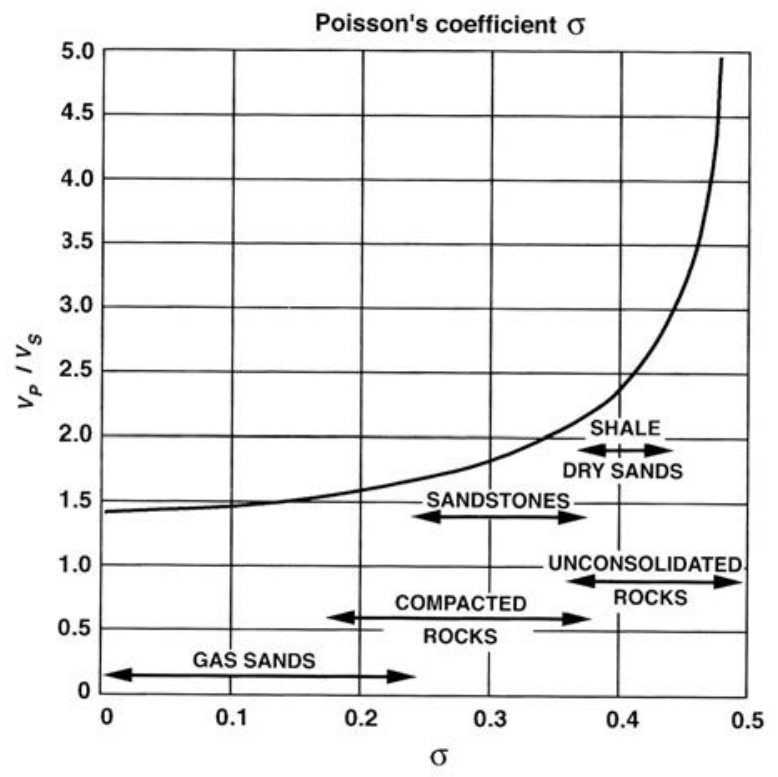

$V_{P} / V_{S}$ ratio as a function of Poisson's coefficient.

Figure 1.2 Poisson's ratio.

In the three-dimensional reference system $(x, y, z)$, the $x$-direction (horizontal) and the z-direction (vertical) represent the vertical plane of the acquisition profile, which contains the source and the receivers and is oriented perpendicular to the main axis of the structure. If the structure has no lateral variation in the y-direction, the structure is said to be cylindrical; variations in displacements along y are zero. On the assumption of a cylindrical structure, the propagation directions of all waves are contained within the plane of the acquisition profile. Particle displacements of the $P$-waves are in the $(\mathrm{x}, \mathrm{z})$ plane. $S$-wave particle motions are either in the plane of the profile - called $S V$-waves (vibrations in the vertical plane) - or perpendicular to the plane of the profile, called $S H$-waves (vibrations in the horizontal plane). The vibration and propagation directions of the various wave types, $P$, $S V$ and $S H$-waves, are illustrated in Figure 1.1-B. 


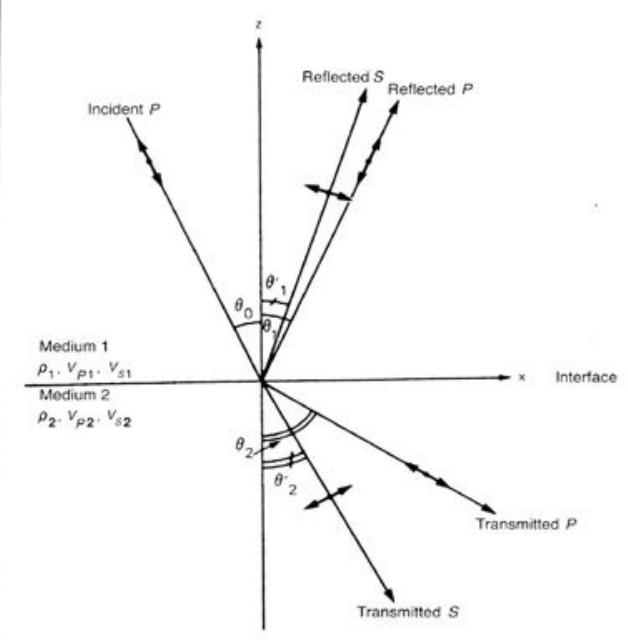

$$
\frac{\sin \theta_{1}}{V_{P 1}}=\frac{\sin \theta_{1}^{\prime}}{V_{S 1}}=\frac{\sin \theta_{2}}{V_{P 2}}=\frac{\sin \theta_{2}^{\prime}}{V_{S 2}}
$$

Types of propagation:

- Reflected waves

- Transmitted waves

- Refracted waves

$$
\left(\theta_{2}=\frac{\pi}{2} \quad \theta_{0}=i_{\mathrm{c}} \quad \sin i_{c}=\frac{V_{i}}{V_{i+1}}\right)
$$

Box 1.1 Snell's law. Angular relationships between incident, reflected and transmitted rays for the various wave types in the case of an incident compressional wave with a wave-front perpendicular to the plane of the diagram. Particle motion is represented schematically (after Lavergne, 1989).

When a $P$ or $S V$-wave strikes an interface at an angle of incidence not equal to zero, four waves are generated: two transmitted (one $P$ and one $S V$-wave) and two reflected (again, one $P$ and one $S V$-wave). The angular relationships between the propagation directions of each of these waves are given by Snell's law (Box 1.1).

The creation of an $S$-wave from a $P$-wave, or vice versa, is a phenomenon called mode conversion. For cylindrical structures, $S H$-waves propagate without mode conversion. Box 1.2 gives the wave equations associated with particle displacements $(\mathrm{u}, \mathrm{v}, \mathrm{w})$ observed in the $(\mathrm{x}, \mathrm{y}, \mathrm{z})$ directions for a seismic profile oriented in the $\mathrm{x}$-direction and perpendicular to the axis of a cylindrical structure on the y-axis.

Particle movements in the y-direction, associated with $S H$-waves, are governed by a simple equation involving only the displacement $\mathrm{v}$ along $\mathrm{y}$ and the velocity $\mathrm{V}_{\mathrm{S}}$, hence there can be no mode conversion (Box 1.2, Eq. (2)). Equations are more complex for the $\mathrm{u}$ and $\mathrm{v}$ displacements associated with the propagation of $P$ and $S V$-waves (Box 1.2, Eq. (1)).

The wave equation may be used to calculate synthetic seismograms that are the response of the subsurface to an excitation. For a distribution of velocities $\left(V_{P}\right.$ and $\left.V_{S}\right)$ and densities $\rho$, a synthetic seismogram can be calculated for a given acquisition geometry. The synthetic seismogram can be compared with an actual field record registered with the same geometric parameters (source and receiver positions). The distribution of velocities and densities can be updated so that an 
optimum fit between the synthetic and the field data is obtained. This procedure is called full waveform inversion (FWI). FWI is discussed in a specific chapter (chapter 5). The wave equation is used in specific processing procedures such as tomography and migration.

$$
\begin{gathered}
\text { Notation: } \quad U_{1}=u(/ / x) \quad U_{2}=v(/ / y) \quad U_{3}=w(/ / z) \quad \frac{\partial}{\partial y}=0 \\
\qquad \begin{array}{c}
\frac{\partial^{2} w}{\partial t^{2}}=\frac{\partial}{\partial x}\left(V_{S}^{2}\left(\frac{\partial w}{\partial x}+\frac{\partial u}{\partial z}\right)\right)+\frac{\partial}{\partial z}\left(V_{P}^{2}\left(\frac{\partial u}{\partial x}+\frac{\partial w}{\partial z}\right)\right)-2 \frac{\partial}{\partial z}\left(V_{S}^{2} \frac{\partial u}{\partial x}\right) \\
\frac{\partial^{2} v}{\partial t^{2}}=\frac{\partial}{\partial x}\left(V_{S}^{2} \frac{\partial v}{\partial x}\right)+\frac{\partial}{\partial z}\left(V_{S}^{2} \frac{\partial v}{\partial z}\right)
\end{array}
\end{gathered}
$$

Equation (1): $P$ - and $S V$-waves with mode conversion

Equation (2): $S H$-waves no mode conversion

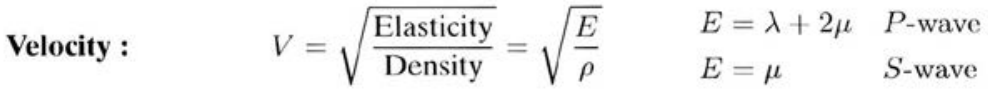

Box 1.2 Wave equation for a 2D seismic profile.

Works such as Achenbach (1973), Dobrin (1988) and Quiblier (1997) should be consulted for more complete information on synthetic seismogram calculation.

\subsection{Seismic methods}

When $P$ or $S$-waves strike the interface at the critical angle $i_{c}$, head waves or refracted waves are generated. This only occurs when a wave perturbation passes from a medium with velocity $V_{i}$ to another with velocity $V_{i+1}$ which is greater than $V_{i}$ and at the critical angle given by $\sin \left(i_{c}\right)=V_{i} / V_{i+1}$.

The critical angle $i_{c}$ is the criterion for differentiating the various seismic methods associated to body wave propagation (Box 1.3):

- $\quad i_{i} i_{c}$ : the method is seismic reflection

- $i=i_{c}$ : the method is seismic refraction

- $\quad i_{i} i_{c}$ : the method is wide angle reflection. In wide angle reflection there is no transmitted energy, only reflected. 


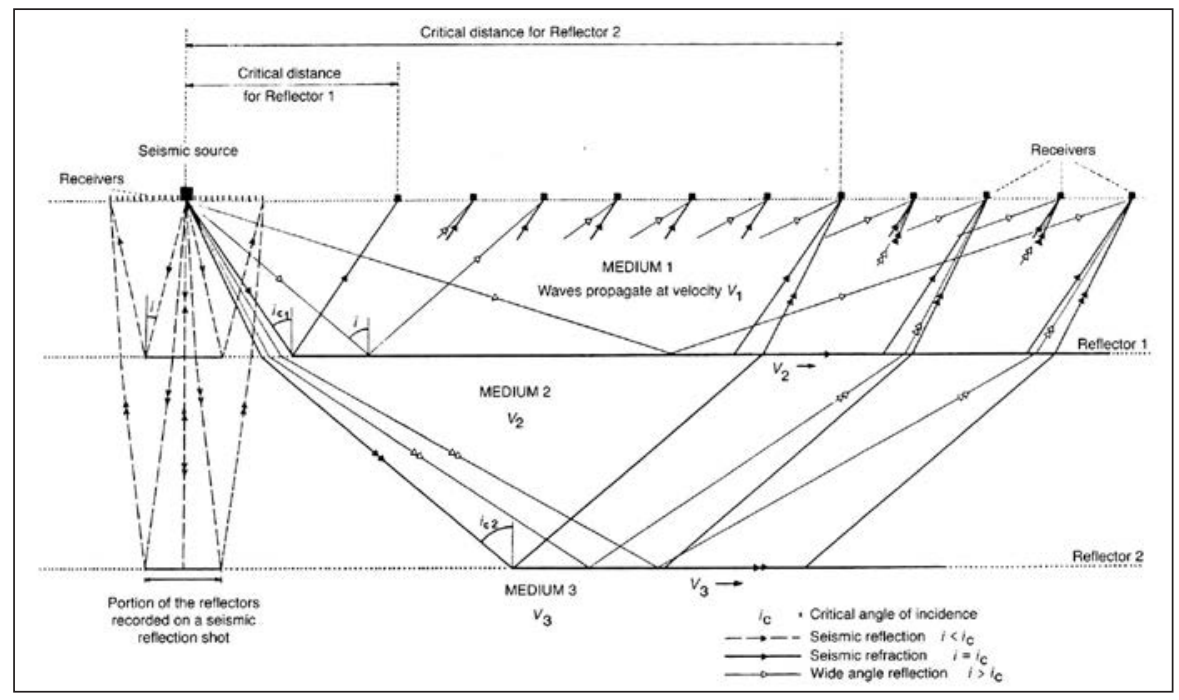

Box 1.3 The various types of seismic surveying. At distances equal or greater than the critical distance, refracted waves or wide-angle reflections can be captured from the reflector.

In addition to the body waves generated by a surface seismic source, which propagate through the subsurface, surface waves also propagate through the earth with their energy concentrated near to the surface. Surface waves, which are mainly sensitive to the shear modulus, do not represent a new wave type; they are interference phenomena resulting from body waves (Figures $1.3-\mathrm{a}, \mathrm{b}$ ). Travelling only within a few seismic wavelengths from the surface of a solid, the lower the frequency of a surface wave, the deeper its penetration depth into the earth. Therefore, in the same medium, waves of different wavelengths affect different depths (Figure 1.3-c).

These waves, which usually exhibit larger amplitudes and lower frequencies than body waves, are used in civil engineering to determine the mechanical parameters (shear velocity and shear modulus) of the first tens of meters below the ground surface.

Among surface waves, which are the main component of ground roll, there are two classes of great interest to characterize the shallow near surface:

a) Rayleigh waves, (R-waves) - travel on the free surface of a semi-infinite solid medium. They are generated from the interference of multiple reflections of $P$ and $S V$-waves. The particle motion has a retrograde elliptical orbit travelling in a vertical plane parallel to the propagation direction, this is polarized in a vertical plane through the propagation direction. The minor axis of the ellipse is parallel to the wave motion direction and equal to two-thirds of its major axis (Figure 1.3-a). On the surface of a solid medium with a Poisson's ratio of $v=0.25$, the Rayleigh waves travel with a velocity $V_{R}$, which is slightly lower than shear wave velocity $V_{S}, V_{R}=0.92 V_{S}$. 
If the semi-infinite medium is overlain by a low-velocity surface layer, as occurs in the field where a weathered layer is present, the elastic constants change with depth, and long wavelengths travel faster than short wavelengths, depending on the elastic properties at greater depths. Such conditions alter some of the wave characteristics and the surface wave is referred to as a pseudo-Rayleigh wave. In this case, the harmonic components of longer wavelengths (lower frequencies) travel faster, and different wavelengths sample different depths in the earth and the propagation velocity can be strongly frequency-dependent (dispersion). The degree of dispersion is a measure of seismic wave velocity as a function of depth and can be used to calculate the thickness of surface layers.

b) Love waves, (L-waves) - develop only if a horizontal low-velocity layer lies over a solid elastic semi-infinite medium. These waves consist of multiples reflections of $S H$-waves while trapped in the horizontal layer. Particle motion is transverse and horizontally polarized (Figure 1.3-b). Since the vertical component does not exist, Love waves are not detected by the vertical geophones. Velocity is close to $S$-wave velocity in the subsurface medium for very long wavelengths; and close to that of the surface layer for short wavelengths. Therefore, these waves always exhibit dispersion and the propagation velocity increases with the period of the harmonic component.
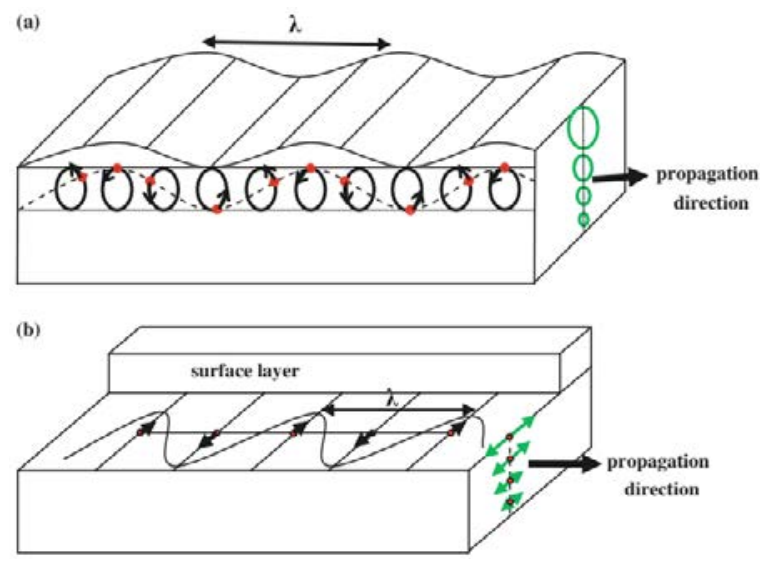

(c)

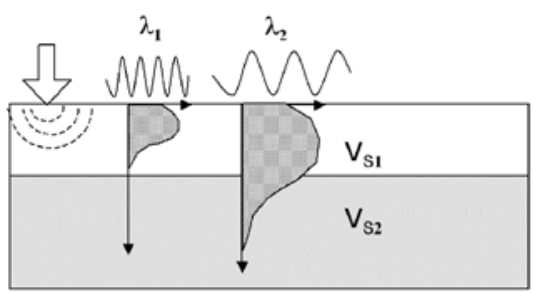

Figure 1.3 A depiction of the displacement induced by plane surface waves. Wavelength ( $\lambda$ ): (a) Rayleigh waves; and (b) Love waves (adapted from Alsadi, 2017); (c) geometrical dispersion of Rayleigh waves associated with short $\left(\lambda_{1}\right)$ and long $\left(\lambda_{2}\right)$ wavelengths. (Adapted from Socco and Strobia, 2004). 
The processing classically applied to surface waves is based on spectral analysis and involves two steps. The first step is the construction of a dispersion curve (a plot of phase-velocity against frequency). A large number of techniques are available, which can be classified into two main classes: frequency-wavenumber or spatial auto-correlation. The next step aims to obtain shear wave velocity (i.e. stiffness) profiles as a function of depth and horizontal position along the seismic survey line. The profiles are calculated using one of the two iterative purposes: matching the experimental dispersion curves to a theoretical curve derived from forward analytical modeling or an automatic least-squares approach.

A field example of surface waves processing is provided in chapter 6 .

\subsection{Example of field records}

A seismic spread is composed of a source spread and a receiver spread. In $2 \mathrm{D}$ seismic surveys, the sources and receivers are located on the same line which defines a $2 \mathrm{D}$ seismic profile. In 3D seismic surveys, the sources and receivers are usually located on 2 orthogonal lines: a line of sources and a line of receivers.

The relative positions of the sources and receivers define the acquisition geometry.

A seismic record is a set of seismic traces recorded at different receiver positions. The seismic trace represents the vibrations of the ground due to wave propagation generated by a seismic source. A geophysicist is able to identify the different seismic waves from a field record. For a simple geological model (a single horizontal layer over an infinite substratum), the arrival times $T$ of the different body waves observed at a distance $\mathrm{X}$ from the source are given by simple T-X equations (T-X curves). Figure 1.4-a shows, for a line of receivers, different possible locations of source points. The distance between 2 geophones on the seismic line is $2 \mathrm{~m}$. If the source point (green dots) is located at the extremities of the receiver spread, the spread is called an end-on spread and the shot is an in-line shot (2D). If the source point (yellow dots) is located on a line perpendicular to the receiver line, the spread is called a cross-spread and the shot is a cross-line shot (3D). The distance between the source position and the receiver position is called the Offset X. An offset can be decomposed in an in-line offset $\mathrm{x}$, and a cross-line offset $\mathrm{y}$, such that:

$$
\mathrm{X}^{2}=\mathrm{x}^{2}+\mathrm{y}^{2}
$$

If $y=0$, and if $x=0$ corresponds to the middle of the line of receivers, the spread is called a split-dip spread, and the shot is a split-dip in-line shot (2D).

Body waves can be differentiated by their T-X curves. Figure 1.4-b shows the ray paths and the T-X curves associated to the direct wave, refracted wave and the reflected wave. The arrival times of a surface wave are locally approximated by linear equations. Figure 1.4-c shows an in-line shot obtained with an end-on spread with the identification of the different waves. Figure 1.5 shows a cross-line shot obtained 
with a cross-line spread. In this figure, the horizontal axis represents the in line offset $\mathrm{x}$, consequently the waves appear with a hyperbolic shape on the T-X diagram.

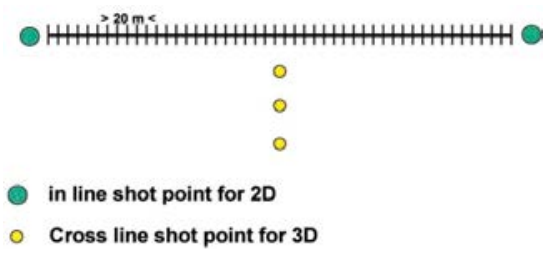

a

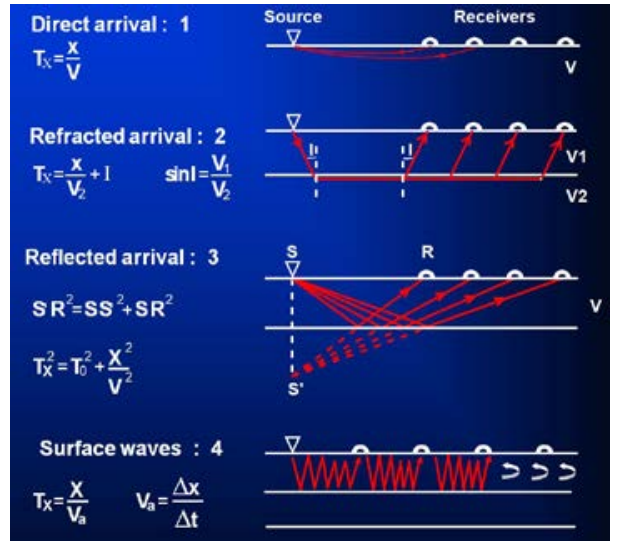

b

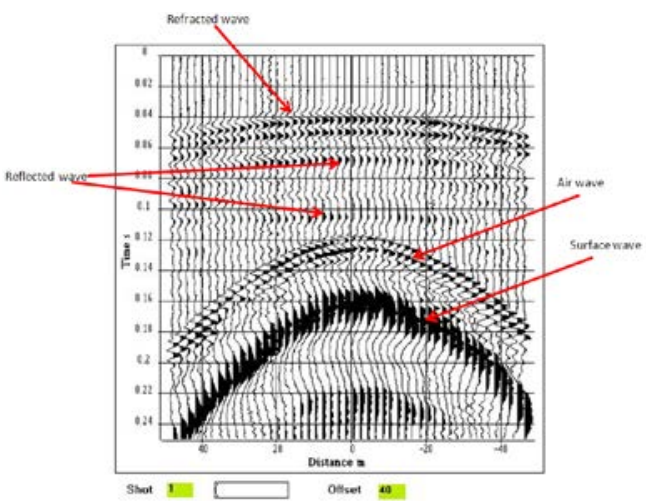

d

Figure 1.4 Seismic spreads (a), T-X curves (b), In-line shot (c), Cross-line shot (d).

Figure 1.5 shows examples of 2D shot points with split-dip spreads (top) and end-on spreads (bottom). For the end-on spread, the 2 shots are obtained with the source located at the 2 extremities of the receiver line. One shot is called a direct shot, the other a reverse shot.

Figure 1.6 shows examples of 3D shot points with cross spread, the cross-line offset varying between 0 and $40 \mathrm{~m}$. With a lateral offset of $40 \mathrm{~m}$, reflected events are clearly visible, as indicated on Figure 1.4-d. 

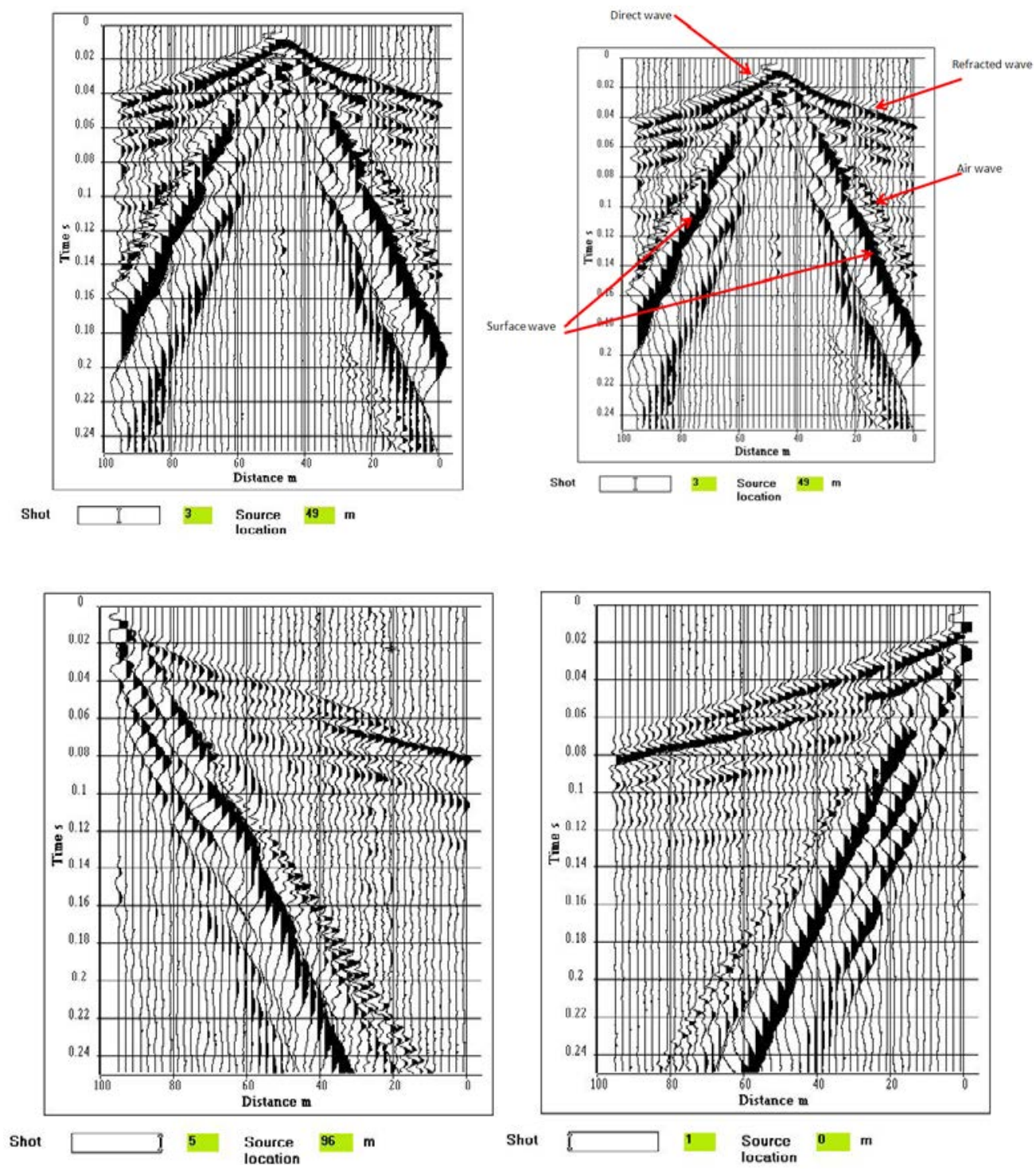

Figure 1.5 2D shot points (top: split dip spread, bottom: direct and reverse end-on spreads). 


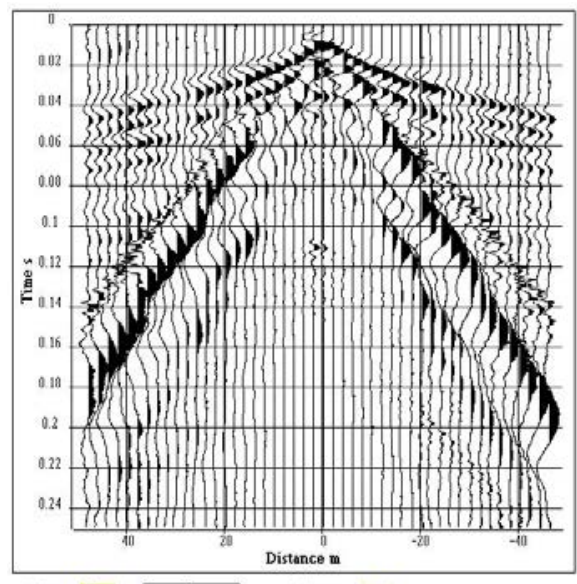

Shot 9

Ollset 0

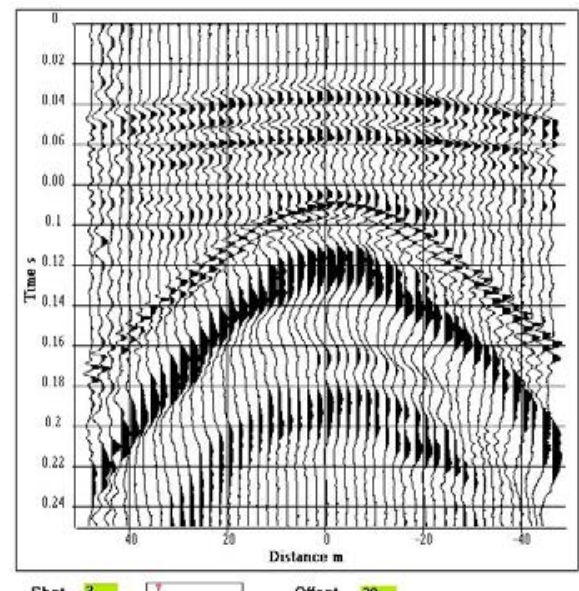

Shot 3 Offret 30
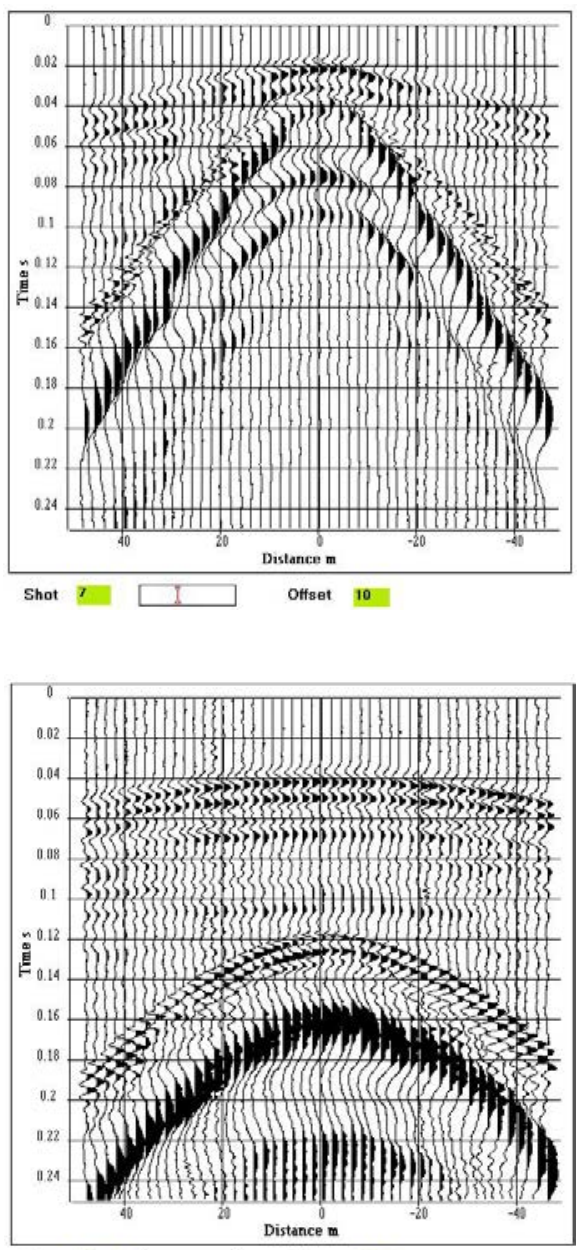

Shat $1 \square$ Ottset

Figure 1.6 3D shot points (cross-spread with cross-line offset ranging from 0 to $40 \mathrm{~m}$ ).

In marine surveys, raw data can be presented in shot point gathers as for land acquisition. Another method of viewing the data is the constant offset section. For each shot, a trace at a given offset is selected. The set of constant offset traces is called a constant offset section. Figure 1.7 is an example of a constant offset section. The first event, named Primary, is the sea bottom reflected wave. The water bottom generates a very characteristic multiple, called a water-bottom multiple (labeled 'Multiple' on the Figure). A multiple reflection is an arrival that has been reflected several times (generally an odd number of times) during its travel path in the subsurface. 
Multiple reflections are produced within the rock layers due to laminations of the rock formations, giving rise to internal multiples.

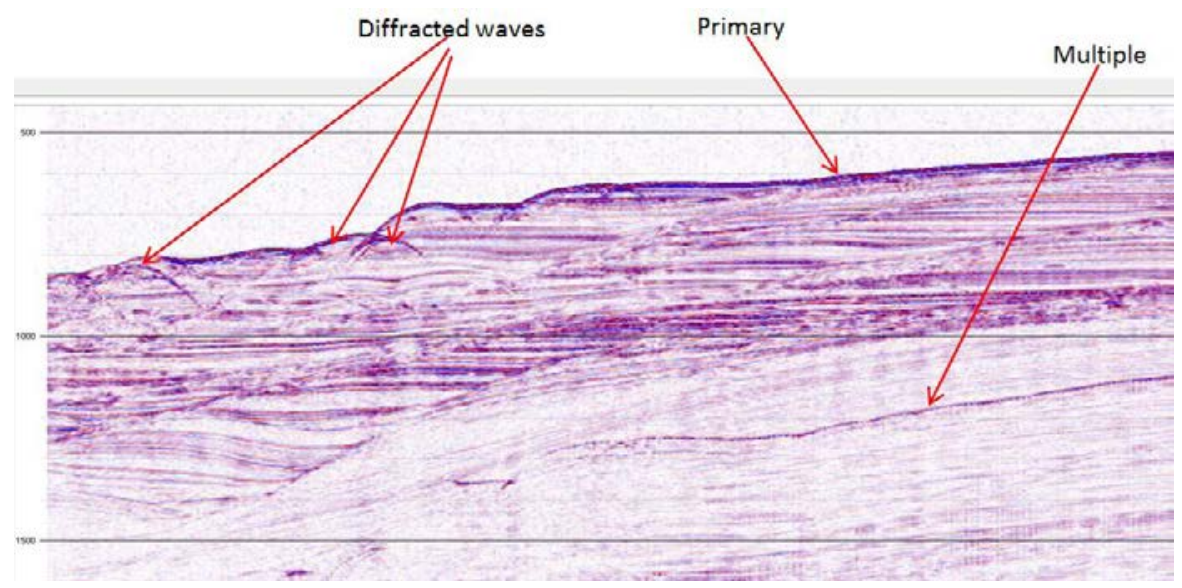

Figure 1.7 Marine constant offset section (data provided courtesy of Craig Fullthorpe, University of Texas).

Other notable spurious arrivals on the records are diffractions. A seismic diffraction is produced when the seismic wave impinges on an angular point or heterogeneity of a size that is less than the wavelength of the incident wave. The heterogeneity or point diffractor radiates energy in all directions. On a seismic record, the point diffractor appears across different traces as a hyperbolic alignment. Figure 1.7 shows diffracted waves due to the heterogeneity of the sea bottom.

\subsection{Wave separation}

Direct and refracted arrivals have linear time-distance curves, reflection events are hyperbolas to a first approximation (Figure 1.4-b). The time-distance curves of surface waves can be treated as locally straight lines. Diffractions have a hyperbolic time-distance curve.

Locally a wave can be characterized by its:

- period $T$ expressed in $\mathrm{s}$ or $\mathrm{ms}$,

- apparent velocity $V a=\Delta x / \Delta t$. The quantity $\Delta t$ is the difference in arrival times of the wave at various receivers at a distance $\Delta x$ apart. 
The wave can be characterized equally well by its:

- frequency $f$, expressed in $\mathrm{Hz}$ (or in cycles/second),

- Wavelength $\lambda$, expressed in $\mathrm{m}$, and

- Wavenumber $k$, expressed in cycles $/$ meter $(\mathrm{c} / \mathrm{m})$.

Frequency is the reciprocal of the period $T(f=1 / T)$. Wavelength is the distance traveled by a wavefront during a time equal to the period $T(\lambda=V T=V / f, V$ being the propagation velocity). Wavenumber $k$ is related to the wavelength $\lambda$ in the same way that frequency $f$ is related to the period $T(k=1 / \lambda)$.

Velocity $\mathrm{V}$ can be expressed in the time-distance domain $(t, x)$ or in the frequency wavenumber domain $(f, k): V=x / t=f / k$.

The translation from the $(t, x)$ domain to the $(f, k)$ domain and vice versa is accomplished by the forward and inverse Fourier transforms. The amplitude representation of a $(t, x)$ record in the $(f, k)$ domain is called a two-dimensional amplitude spectrum.

Waves can be separated by different filters (Mari, 2015) such as:

- Frequency filter $(f)$,

- Wavenumber filter $(k)$,

- Apparent velocity filter in the $(f, k)$ domain $(V a=f / k)$.

Other filters can also be used, such as:

- SVD (Singular Value Decomposition) filter,

- polarization filter,

- ...

Figure 1.8 shows an example of an in-line shot point obtained with an impulse source. The receiver spread is composed of 48 single geophones. The distance between 2 adjacent geophones is $5 \mathrm{~m}$. The time sampling interval is $0.25 \mathrm{~ms}$ and the recording length is $0.5 \mathrm{~s}$. The data are presented both in the time distance domain and in the frequency - wavenumber domain. The wave separation is done in the $(f, k)$ domain by apparent velocity filters.

The $2 \mathrm{D}$ amplitude spectrum of the raw data (Figure 1.8-a) shows that some of the energy is located in the negative wavenumbers. The waves associated with this energy propagate with negative apparent velocities.

The main energetic wave fields are the direct waves, and the low and high apparent velocity pseudo-Rayleigh waves (Figures 1.8-b and c). Figure 1.8-d shows the wavefield associated with negative wavenumbers. These waves are converted refracted waves. The refracted wave is shown in Figure 1.8-e. The reflected waves, shown in Figure 1.8-f, are characterized by small wavenumbers and, consequently, very high apparent velocities. 


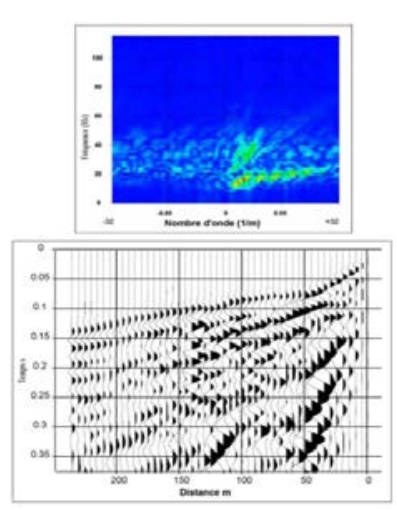

a

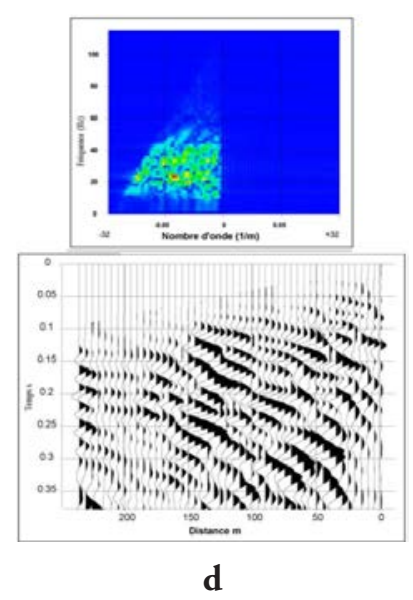

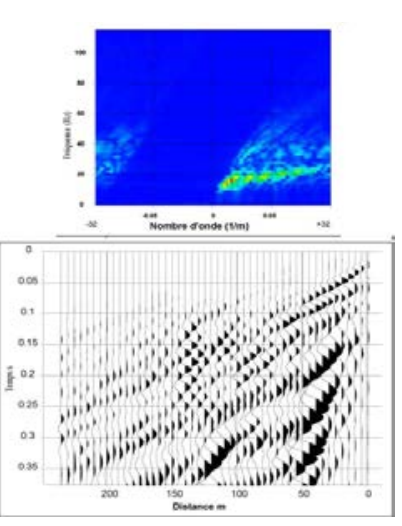

b
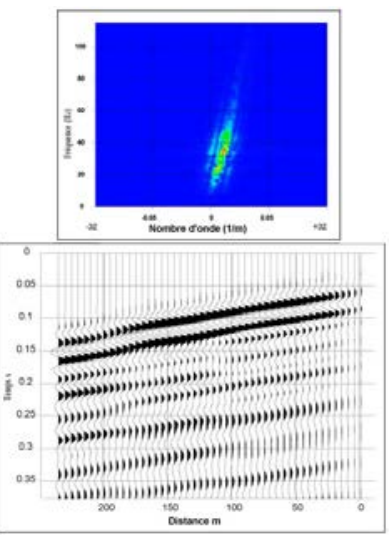

e

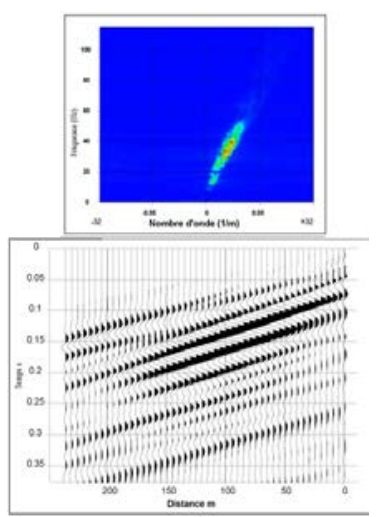

c

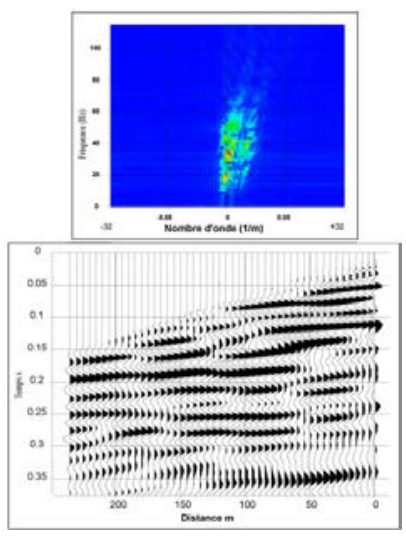

f

Figure 1.8 wave separation - In line shot point (a) raw data, (b) direct wave and slow Rayleigh wave, (c) fast Rayleigh wave, (d) converted refracted waves, (e) refracted wave, (f) reflected waves (Normalised displays).

Figure 1.9 is an example of wave separation by wavenumber filters. The example shown is based on a VSP (Vertical Seismic Profile; Mari and Vergniault, 2018) data set. From top to bottom, this figure shows:

- Raw data,

- Downgoing wavefield associated with positive wavenumbers,

- Upgoing wavefield associated with negative wavenumbers. 


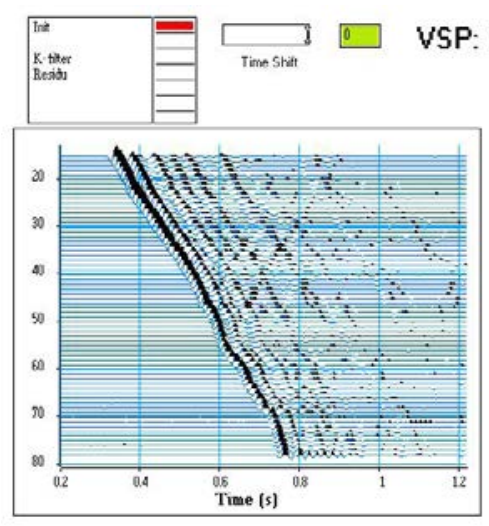

SP: K-Filter GRAPHE
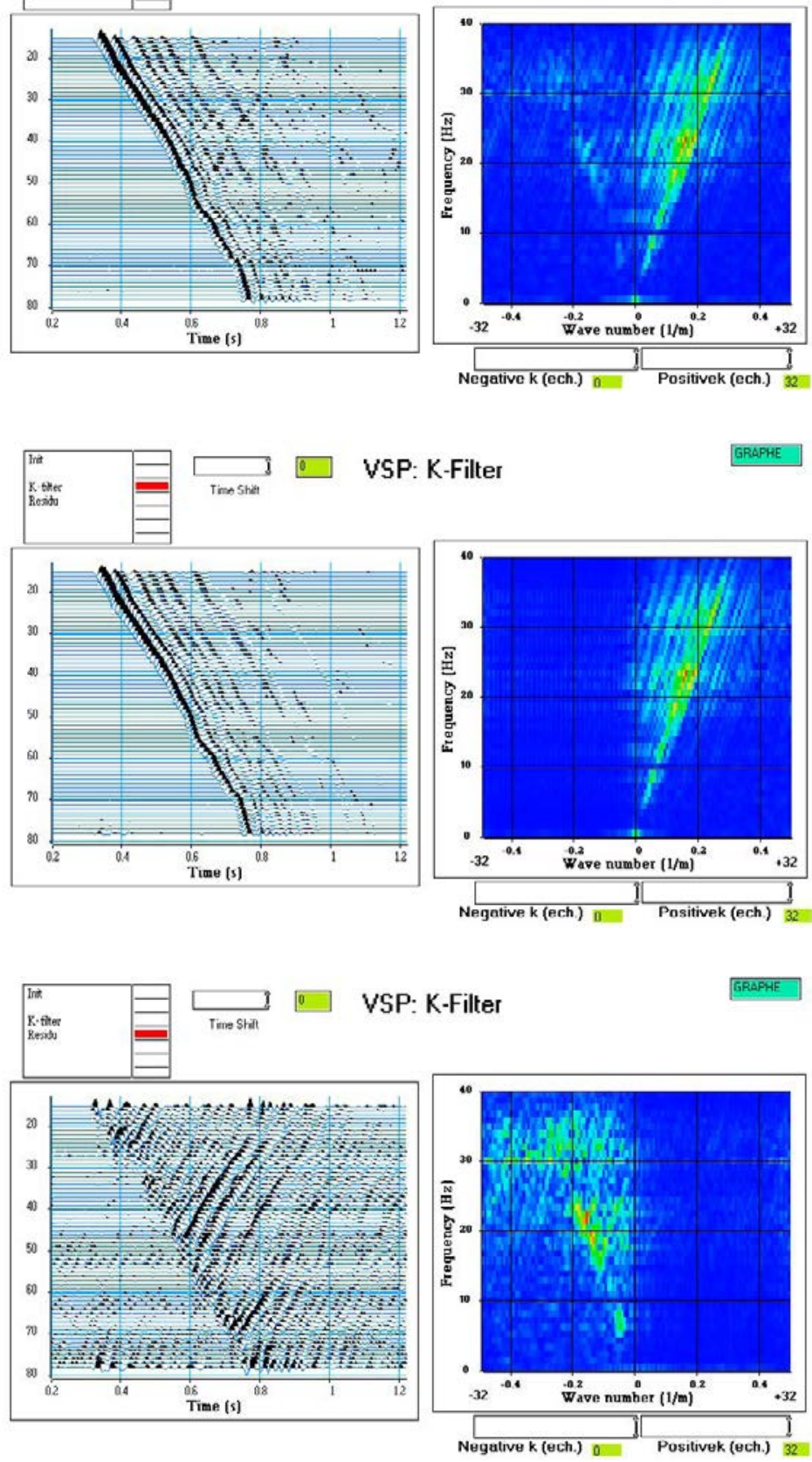

Figure 1.9 wave separation - VSP data. From top to bottom: raw data, downgoing wavefield, upgoing wavefield. 


\subsection{Conclusion}

In summary, a seismic record contains:

- direct arrivals,

- refracted waves,

- surface waves,

- reflected waves,

- diffractions,

- multiples, and

- noise unrelated to the seismic shot.

The various waves can be categorized according to their characteristic properties. One of the most distinguishing properties is a wave's time-distance curve. The acquisition geometry and the type of seismic survey must be taken into consideration. The waves can be separated by filters such as $(f, k)$ filters in the $(f, k)$ domain.

\section{References}

Achenbach J.D., 1973, Wave propagation in Elastic solids, North Holland Publishing Company, $3^{\text {rd }} \mathrm{Ed}$.

Alsadi H.N., 2017, Seismic hydrocarbon exploration, 2D and 3D techniques. Springer, ISBN: 978-3-319-40435-6. http://www.springer.com/978-3-319-40435-6.

Dobrin M.B., Savit C.H., 1988, Introduction to Geophysical Prospecting, $4^{\text {th }}$ edition McGraw-Hill Book Co., 867 p.

Ensley R., 1985, Evaluation of direct hydrocarbon indicators through comparison of compressional- and shear-wave seismic data: a case study of the Myrnam gas field, Alberta, January Geophysics 50(1), DOI: 10.1190/1.1441834.

Lavergne M., 1989, Seismic methods, Éditions Technip, Paris, ISBN: 2-7108-0552-9.

Mari J.L., 2015, Signal processing for geologists \& geophysicists, e-book, DOI:10.2516/ ifpen/2011002. http://books.ifpenergiesnouvelles.fr/ebooks/signal-processing/

Mari J.L., Vergniault C., 2018, Well seismic surveying and acoustic logging, EDP Sciences, DOI: 10.10051/978-2-7598-2263-8, ISBN (ebook): 978-2-7598-2263-8. https:// www.edp-open.org/well-seismic-surveying-and-acoustic-logging

Socco L.V., Strobbia C., 2004, Surface-wave method for near-surface characterization: a tutorial, Near Surface Geophysics, 165-185.

Quiblier J., 1997, Propagation des ondes en géophysique et en géotechnique. Modélisation par méthodes de Fourier, Éditions Technip, Paris. 